\title{
Machine learning for multi-dimensional optimisation and predictive visualisation of laser machining
}

\author{
Michael D. T. McDonnell ${ }^{1}$ (D) Daniel Arnaldo ${ }^{2} \cdot$ Etienne Pelletier ${ }^{2} \cdot$ James A. Grant-Jacob $^{1} \cdot$ Matthew Praeger $^{1}$. \\ Dimitris Karnakis ${ }^{2} \cdot$ Robert W. Eason ${ }^{1} \cdot$ Ben Mills $^{1}$
}

Received: 11 February 2020 / Accepted: 13 November 2020 / Published online: 11 January 2021

(c) The Author(s) 2021

\begin{abstract}
Interactions between light and matter during short-pulse laser materials processing are highly nonlinear, and hence acutely sensitive to laser parameters such as the pulse energy, repetition rate, and number of pulses used. Due to this complexity, simulation approaches based on calculation of the underlying physical principles can often only provide a qualitative understanding of the inter-relationships between these parameters. An alternative approach such as parameter optimisation, often requires a systematic and hence time-consuming experimental exploration over the available parameter space. Here, we apply neural networks for parameter optimisation and for predictive visualisation of expected outcomes in laser surface texturing with blind vias for tribology control applications. Critically, this method greatly reduces the amount of experimental laser machining data that is needed and associated development time, without negatively impacting accuracy or performance. The techniques presented here could be applied in a wide range of fields and have the potential to significantly reduce the time, and the costs associated with laser process optimisation.
\end{abstract}

Keywords Laser machining $\cdot$ Neural networks $\cdot$ Deep learning $\cdot$ Fabrication $\cdot$ Manufacturing $\cdot$ Micro-structuring

\section{Introduction}

Femtosecond lasers allow material processing with extremely high precision (Mills et al. 2014; Misawa et al. 2000; Momma et al. 1997), due to the relatively small heat affected zone from each pulse (Le Harzic et al. 2002). However, nanosecond lasers offer a more cost-effective solution and higher material removal rates (Neuenschwander et al. 2013; Ren et al. 2005). Although exact cost differences are challenging to determine, both due the unique set-up requirements and developments in technology, the number of photons produced per second per dollar cost has historically been higher for nanosecond, as compared to femtosecond, lasers. Any method that allows a nanosecond laser to achieve machining quality that is comparable with that of femtosecond systems would be highly desirable as it offers a route to greatly reduce manufacturing costs.

Michael D. T. McDonnell

m.d.t.mcdonnell@soton.ac.uk

1 Optoelectronics Research Centre, University of Southampton, Southampton SO171BJ, UK

2 Oxford Lasers, Oxford OX117HP, UK
Many laser machining tasks that are currently carried out on femtosecond systems could, in principle, be achieved with nanosecond lasers. However, due to the nonlinear dependence on laser machining parameters (pulse energy, repetition rate, number of pulses (Cheng et al. 2009; GrantJacob et al. 2014; Lorbeer et al. 2017)) this may require extremely precise and repeatable parameter control. The domain of parameters where such high-quality machining can be achieved on a nanosecond system may be very narrow, and hence very challenging to identify without a detailed systemic experimental exploration. Nevertheless, the potential cost savings mean that for any given laser machining task, it is worth exploring the feasibility of using nanosecond pulses before deciding that femtosecond machining is required.

Given that there may be multiple laser parameters, a systematic experimental exploration can be very timeconsuming, and hence not feasible. For example, 3 investigated laser parameters, with 10 possible different values each, corresponds to 1000 total measurements, extending to 1 million combinations if there were instead 100 possibilities for each input value. In addition, this process would have to be repeated for any change in laser or material conditions, for example laser beam quality, or a different material type. 
A systematic experimental approach may be forced then to discard certain regions of the parameter space, introducing the risk of human bias. Therefore, a systematic experimental approach may tend to eliminate high laser pulse energies, high laser frequencies or a combination of both from the parameter space. However, it will be shown here that the use of high repetition rates is in fact required to achieve the desired machining quality with nanosecond pulses.

One method of finding optimal process parameters for any experiment is the gradient search method, where the mismatch between actual machining quality and desired machining quality is minimised through changing the value of one parameter at a time. This will certainly lead to a minimum, but due to the nonlinear nature of the parameter space, is extremely unlikely to be a global minimum. In addition, if there are only very few possible parameter combinations that will offer the required quality, then such a solution is unlikely to be found by a gradient search method, and instead a systematic experimental exploration will still be needed.

Alternatively, if the entire laser machining process could be modelled (i.e. starting from equations that accurately describe all interactions between light and matter) then the optimal parameter configuration could theoretically be determined directly, without the need for any experimental optimisation. Unfortunately this approach is generally not effective, as many approximations have to be made to simplify such modelling and simulations of light-matter interaction rapidly become computationally intractable when the model size is increased to represent experimentally useful scale dimensions (Mazhukin 2017). It is also challenging to include subtle effects such as imperfections in the beam quality or sample surface in such a simulation (Otto et al. 2012). Furthermore, to achieve sufficient accuracy it may be necessary that the model incorporates complexities such as chemical reactions, plasma generation, and multiphoton laser interactions along with many more; typically, this is not possible and in reality, some important contributing processes may even be unknown.

A novel alternative to all above, and one that is rapidly gaining traction across academia and industry, is the application of neural networks to produce a simulation of a complex system directly from a small experimental dataset (Feng et al. 2019; Zhang and Ling 2018). This approach has been shown to be extremely effective at simulating laser machining (Heath et al. 2018; Heath et al. 2018; Mills et al. 2018), and is considerably faster in terms of computation speed. Neural networks have demonstrated the capability to learn and simulate physical phenomena such as the optical diffraction limit directly from experimental data and without the need for inclusion of any equations that describe the underlying physics (Mills et al. 2018). Artificial neural networks (ANNs) are therefore an attractive method for simulating laser machining. One particular advantage is that any experi- mental subtleties that are present in the training data, such as inhomogeneities in the laser beam shape, are automatically included in the simulation.

ANNs are an area of machine learning inspired by biological neural networks. Fundamentally, they consist of an input, an output, and some number of hidden layers. These layers are of nodes which are referred to as neurons. In an ANN the input and output is a vector, with each hidden layer consisting of neurons that each act as a non-linear function acting on all inputs. In general, ANNs are fully connected, meaning that each neuron in one layer is connected to each neuron in the next layer, all the way until the final layer. The variables associated with each neuron of an ANN are generally randomly initialised and later automatically updated (referred as training the network) by a technique knowns as backpropagation (Werbos 1974). This process uses known input-output training pairs, where the ANN sequentially processes a training input and generates an output, which is then compared to the associated output training data component. The difference between the generated and the real output is determined, and the ANN parameters are automatically modified accordingly. A loss function, such as mean squared error, is often used to measure this performance. The mathematics behind ANNs and their training can be found in the book 'Deep Learning' by Goodfellow et al. (2016).

Previous work in this field generated a model that relates dimple depth and diameter to the pulse energy used (Yousef et al. 2003). In this work one ANN takes the numerical values for the dimple depth and diameter as inputs and predicts the pulse energy needed to achieve this. A second ANN then takes the same inputs as the first, along with the predicted pulse energy and then outputs the expected standard deviation in both depth and diameter. Casalino et al. (2017) and Campanelli et al. (2013) also show good applications of ANNs to determine machining characteristics, although the parameter space used was small and each ANN was used to predict a single parameter. Teixidor et al. (2015) investigated the performance of a wide range of machine learning techniques mainly focussing on discrete variables rather than continuous. Two additional machine learning methods were also investigated as part of this experiment: the Gaussian process and support vector machines. These techniques were found to provide inferior results, and the relative accuracies of the three techniques will be discussed alongside the machine learning methodology.

Neural networks have also been used to monitor image based processes using a style of network called convolutional neural networks (CNNs). Gonzalez-Val et al. (2020) performed experiments in laser metal deposition and welding to determine machining quality via two different metrics. They determined that it would be possible to create in in line defect detection system, allowing for early correction of errors. Kim et al. (2018) performed a more general investigation into 
smart manufacturing as a field. While very high level, their work found ANNs and support vector machines to account for the most promising areas as long as care is taken with the input parameters. Penumuru et al. (2020) also looked into how machine learning could be incorporated into the manufacturing paradigm of industry 4.0 (Mittal et al. 2019). Their work looked at a material classification problem and compared several methods including support vector machines and logistic regression, along with the VGG-16 deep CNN (Simonyan and Zisserman 2015). Out of the methods tested they found that support vector machines performed best, with the deep neural network being too computationally intense.

The use of neural networks has also been extended to other areas of laser manufacturing, such as laser welding (Asif et al. 2020; Günther et al. 2014, 2016), additive manufacturing (Li et al. 2020; Mahato et al. 2020; Mycroft et al. 2020), and a method to reconstruct laser pulses (Zahavy et al. 2018). Here, we extend on both these, and previous (Arnaldo et al. 2018), works in the area of laser surface texturing.

Here we demonstrate the application of neural networks for identification of the optimal laser parameters for machining microscale blind holes (referred to here as dimples). Our motivation for this paper is to firstly show that the neural network predictions are accurate (which we demonstrate comprehensively in Table 2 and Fig. 4) and secondly to explore the methodology for detailed investigation of the neural network predictions and to illustrate the flexibility of this approach, an example of which can be seen in Fig. 6. We also demonstrate the ability of generative neural networks to generate surface profiles that can recreate the height map of the experimental results, allowing for further investigation. This manuscript provides, for the first time, a comparison between ANN and GAN modelling approaches for the optimisation of laser machining, highlighting the capabilities of each technique and contrasting these with the conventional approach of making a complete, systematic, experimental parameter sweep. There are two performance criteria that each dimple must meet, which relate to the depth of the crater and the height of redeposited material, referred to here as the 'crown' height. Each dimple crater must be deeper than $4 \mu \mathrm{m}$, and the crown height should be minimised.

Arrays of dimples, with minimised crowns, are widely used to reduce surface friction, minimise wear and therefore extend lifetime of critical mechanical components (engine cylinder liners, pistons, bearings, etc.) (Mezzapesa et al. 2013; Qu et al. 2014; Sakai et al. 2007; Scaraggi et al. 2014). Fabrication of such surfaces is relatively simpler when using femtosecond lasers, due to the considerably reduced heataffected zone (Sakai et al. 2007). Here, we show that neural networks can provide the methodology for identification of the particular laser parameters that enable the fabrication of anti-friction surfaces using nanosecond pulses, despite the fact that they offer considerably less precision and a larger heat-affected zone. Throughout this manuscript, the terminology used will adhere to the standards set out by Stegemann and Buenfeld (1999). This states that the data used to evaluate the network performance during training but not used to update weights is the validation set. The test dataset is held separate from this and used for comparison once the network had been fully trained, and in this case was held by Oxford Lasers.

\section{Experimental methods}

The proposed methodology was to collect laser machining data for a limited selection of laser parameters, use this data to train a neural network, and then to query the neural network in order to identify the optimal parameters for meeting the criteria for laser machining dimples that are deeper than $4 \mu \mathrm{m}$ and which have a minimised crown.

\section{Experimental data collection}

The material chosen for this experiment was grey cast iron DIN GG20 (ATSM A48 n.30) with 20\% max ferrite phase (the remainder being pearlite phase) as it exhibits favourable tribological and machining properties (Oloyede et al. 2016). The samples were laser machined using an ytterbium fibre laser, $\mathrm{M}^{2}<1.8$, operating at a central wavelength of $1060 \mathrm{~nm}$ and with a pulse duration of $0.17 \mathrm{~ns}$. The laser beam passed through a beam reducer and was focused down to a spot size of $36 \mu \mathrm{m}$ diameter (at $1 / \mathrm{e}^{2}$ value of maximum intensity as measured using the $\mathrm{D}^{2}$ method (Liu 1982)) using a lens with a focal length of $100 \mathrm{~mm}$. The material itself was formed into cylindrical sections cut into $30^{\circ}$ segments. 5-axes stages accurate to $2 \mu \mathrm{m}$ were used to control machining of the inner surface of the cylindrical segment. There was also control of the optical axis via galvanometric mirrors combined with $\mathrm{f}$ theta lenses. After the dimples were machined, the resultant surface profile was supplied as a 2D array of heights constituting a height map. The set-up is shown in full in Fig. 1.

The laser repetition rate was adjusted in the range 10 to $1200 \mathrm{kHz}$ (maximum output frequency possible), the laser pulse energy from 5 to $50 \mu \mathrm{J}$, and the number of incident laser pulses on target was chosen to be between 50 and 400 . These values are summarised int Table 1. For each input parameter combination, there were up to 5 associated 3D profiles. Due to random fluctuations in experimental conditions, such as those associated with laser pulse energy and beam quality, each of the output profiles were slightly different. The training data therefore included cases where the same input parameters corresponded to different output profiles. Topographies of the resulting laser-machined surfaces were measured via confocal microscopy and some example 


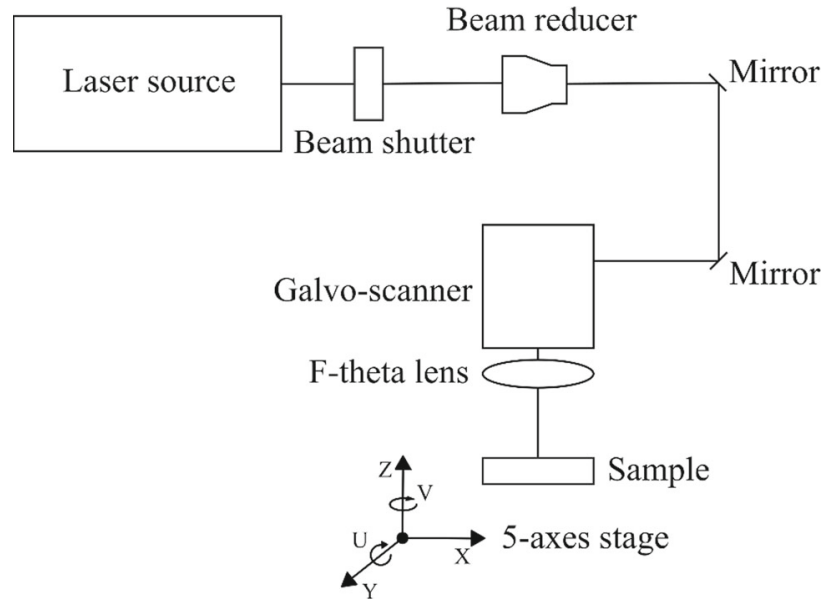

Fig. 1 Laser machining set-up

Table 1 Full range of parameters for investigation

\begin{tabular}{lcc}
\hline & Minimum value & Maximum value \\
\hline Pulse energy $(\mu \mathrm{J})$ & 5 & 50 \\
Number of pulses & 50 & 400 \\
Repetition rate $(\mathrm{kHz})$ & 10 & 1200 \\
\hline
\end{tabular}

data is shown in Fig. 2. This gave 170 total combinations with 884 individual dimples, 155 of the combinations were then randomly chosen to form the training set, with the remainder being the validation set.

Although the network was trained on each individual data point, with multiple $3 \mathrm{D}$ profiles per parameter combination, some contaminated data was removed. An example of such contamination includes areas with considerable external debris where the recorded height did not accurately match the height of the crown. This, in combination with the fact that that there were multiple measurements taken for each parameter combination meant that the effect of non-Gaussian noises, which can cause issues (Stojanovic and Filipovic 2014; Stojanovic and Nedic 2016) was minimised.

\section{Machine learning methodology}

The ANN used in this work was constructed using a series of three identical blocks followed by two dense layers (also known as fully connected layers) and a final activation layer as can be seen in Fig. 3. Each of the dense blocks consisted of a dense layer with 256 neurons and a leaky ReLU activation layer. This was then followed by a $50 \%$ dropout layer and a batch normalisation layer. The output layer consisted of a further dense layer with 256 neurons and leaky ReLU activation. A dense layer with 2 neurons and a ReLU activation that formed the output from the network which was optimised using the Adam optimiser (Kingma and Ba 2014). The learning rate was chosen specifically in order to ensure a balance between convergence speed and accuracy. As absolute speed of training wasn't the primary goal, a lower than average learning rate of $1 \mathrm{e}-5$ was chosen. The output from the neural network was a two-value vector containing crown height and dimple depth measured in microns. The crown height was defined as the 99th percentile of the height map, whilst dimple depth was defined as the 1st percentile of the height map. Removed volume is defined as the total volume that is enclosed by the plane where height $=0 \mu \mathrm{m}$ (the surface prior to laser machining) and the inner surface of the dimple.

Training of the neural network took approximately $5 \mathrm{~min}$. In order to determine the predictive capability of the neural network, 18 of the 155 original parameter combinations were withheld from the training process for later use as a validation data set. Once trained, the neural network was used to predict the crown height for combinations of laser parameters that corresponded to data in the validation data set.

A comparison of the neural network prediction for crown height, along with the matching experimental data from the validation data set, is shown in Fig. 4a. In the majority of cases, the predicted crown height is very close to the experimentally observed value. Of particular interest to this work is how the prediction accuracy varies as a function of the number of experimental data points in the training dataset. This relationship is shown in Fig. 4b, where subsets of different sizes were produced by randomly selecting the required number of samples from the training data pool. When the training subset contains a high number of parameter combinations strong agreement with the validation data is shown, with errors as low as $13 \%$. This is comparable to the standard deviation of the whole experimental dataset at $12.8 \%$, hence demonstrating that the neural network had indeed encapsulated the multidimensional trends present in the experimental data. Interestingly, while it is well known that generally more training data would result in higher accuracy this plot suggests that might not always be the case. In fact, if a $20 \%$ error in NN prediction is considered acceptable for the laser machining process, then a dataset that contains only 70 parameter combinations is adequate and can be as effective as one containing almost twice that number. When evaluating the efficacy of a reduced number of training data combinations, a randomly chosen subset of the complete training data set was used for training, and therefore all data on the figure corresponds to a single experiment. This meant that each data point from each chosen combination was seen by the network during training, leading to approximately 5 times as many data points as parameter combinations. Crucially, due to the random nature of the subset of data chosen, the 70 combinations will have had a similar range to the origi- 

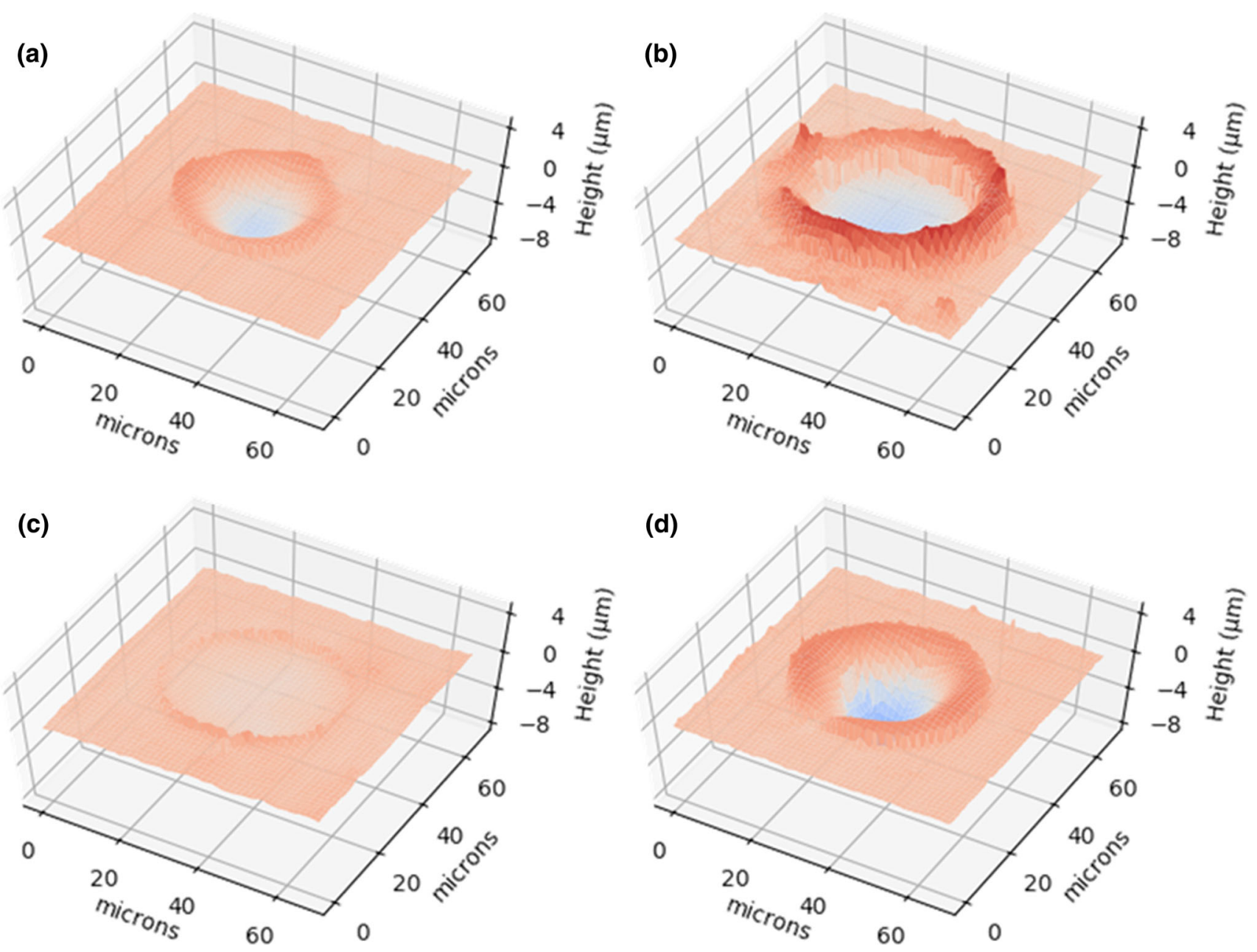

Fig. 2 Example surface profiles on grey cast iron for a range of laser machining parameters. a repetition rate of $10 \mathrm{kHz}$, pulse energy $47.17 \mu \mathrm{J}$ and 200 pulses. b $1200 \mathrm{kHz}, 12.41 \mu \mathrm{J}$ and 100 pulses. c $100 \mathrm{kHz}, 38.67 \mu \mathrm{J}$ and 50 pulses. $\mathbf{d} 1000 \mathrm{kHz}, 26.80 \mu \mathrm{J}$ and 50 pulses

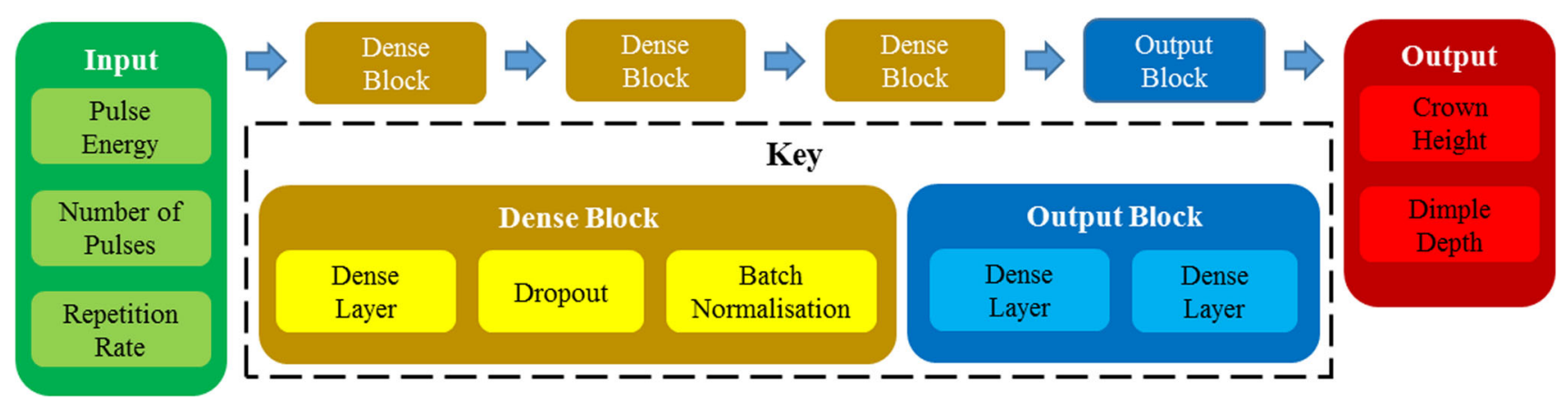

Fig. 3 Block schematic of the ANN

nal data, allowing the network to train over the full range of inputs.

Both the Gaussian process and support vector machines were investigated in order to draw a comparison with the ANN for this task. For the Gaussian process a single model was used to predict both the crown height and the depth. The best performing kernels were the radial-basis function (RBF) and rational quadratic kernels with mean errors of $36.1 \%$ and $32.0 \%$ respectively when predicting the crown height of the validation dataset. The support vector machine method performed better, although required two separate models to predict both the crown height and depth. The best performing kernel was the RBF kernel with a mean error of $24.4 \%$ when predicting the crown height of the validation dataset. In 
Fig. 4 Performance of the ANN. a Comparison of predicted crown height with experimentally measured values from the validation data set. b Predictive capability of the neural network for varying training data set size
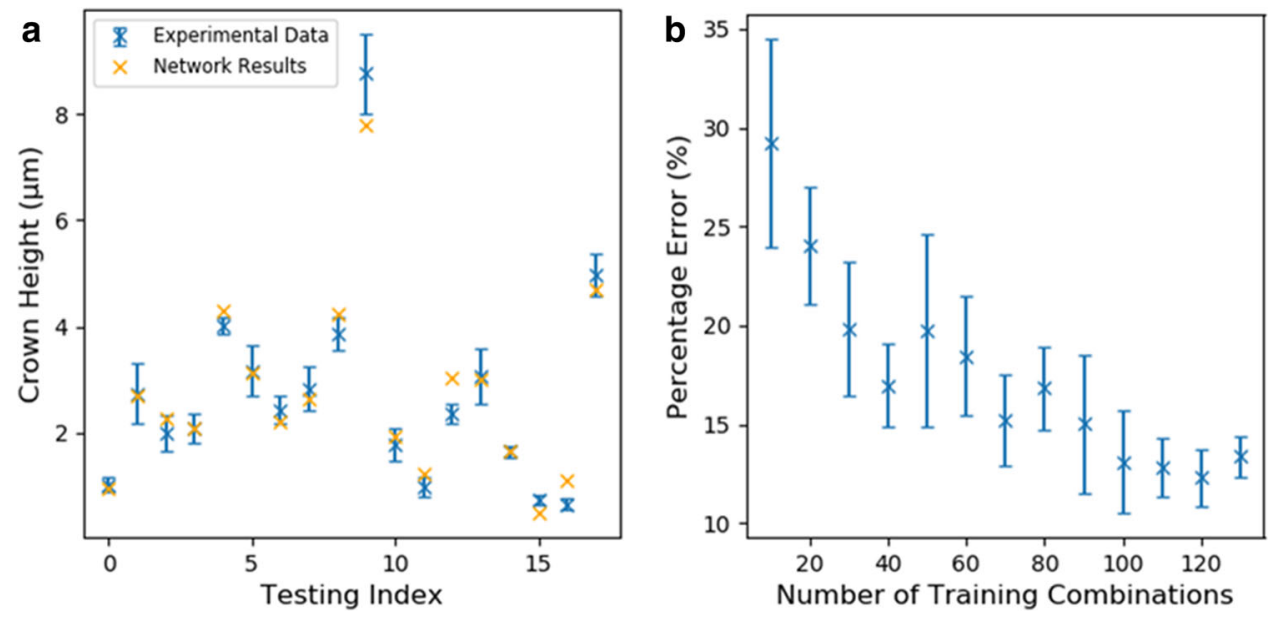

Table 2 Comparison of machine learning techniques

\begin{tabular}{lllll}
\hline & Neural network & Gaussian process & Rational quadratic & $\begin{array}{l}\text { Support vector } \\
\text { machine }\end{array}$ \\
\cline { 2 - 4 } & $\begin{array}{l}\text { Fully connected } \\
\text { network }\end{array}$ & $\begin{array}{l}\text { Radial basis } \\
\text { function }\end{array}$ & $\begin{array}{l}\text { Radial basis } \\
\text { function }\end{array}$ \\
\hline Mean percent error & 13.0 & 36.1 & 32.0 & 24.4 \\
\hline
\end{tabular}

addition to the higher error, the model also predicted several negative values for depth and crown height. These results are summarised in Table 2 and indicate that the ANN provided superior accuracy for the prediction of the crown height of the machined dimples.

In general, neural networks are only considered to be suitable for making predictions that require interpolation between the training data values, as they can have undefined behaviour for extrapolation outside of these bounds. For this dataset, if, rather than having to meet a minimum depth, the only requirement was to minimise the crown height, the neural network inferred that all of the parameters (repetition rate, pulse energy, and number of pulses) should be minimised to their smallest possible values. Of course, this does indeed result in minimisation of the crown height, since no laser machining will occur but is not a particularly helpful outcome. This result reinforces the fact that the criteria for optimisation must be carefully selected and accurately defined.

\section{Justification for ANN structure}

There are many hyper parameters (such as learning rate, number of training epochs, and network size) that can be adjusted to fine tune ANN performance and hence improve their speed and accuracy. In general, larger networks will have a better final accuracy, although they will often take longer to train and may result in overfitting on small datasets. To ensure that the ANN used was suitable for the dataset, ANNs of dif- ferent sizes were trained for a fixed number of epochs, and the accuracy investigated. Figure 5 shows the results from this investigation, with the percentage error of the validation data displayed against the number of neurons in each layer of the network. As expected, as the number of neurons was increased, the percentage error decreased nonlinearly, with a network with 512 neurons only being $0.8 \%$ more accurate than one with 256. At 512 neurons per layer there was also small evidence of overfitting as the validation loss increased to $0.5 \%$ above that for the 256 neuron network. Regarding the time required for training, the network size was not usually the limiting factor to the time required to train, with the 512 network taking 5 min $4 \mathrm{~s}$, with the others taking approximately $4 \mathrm{~min} 40 \mathrm{~s}$. Due to these factors the network was chosen to have 256 neurons per layer.

During this test each size of ANN was trained 10 times using unique random initialisations for each training run, proving the error in the results seen in Fig. 4. When using only 10 neurons per layer the standard deviation in the final error of the network was $5.8 \%$, reducing to $1.1 \%$ for the 256 neuron network used. This shows that performance of the network used was largely independent of the initialisation state of the network, particularly as the size of the network was increased.

\section{Parameter optimisation by neural network}

In traditional optimisation tasks, many complex algorithms are employed (Desiré et al. 1978) such as ones inspired 
Fig. 5 Percentage error of the ANN for varying numbers of neurons per layer

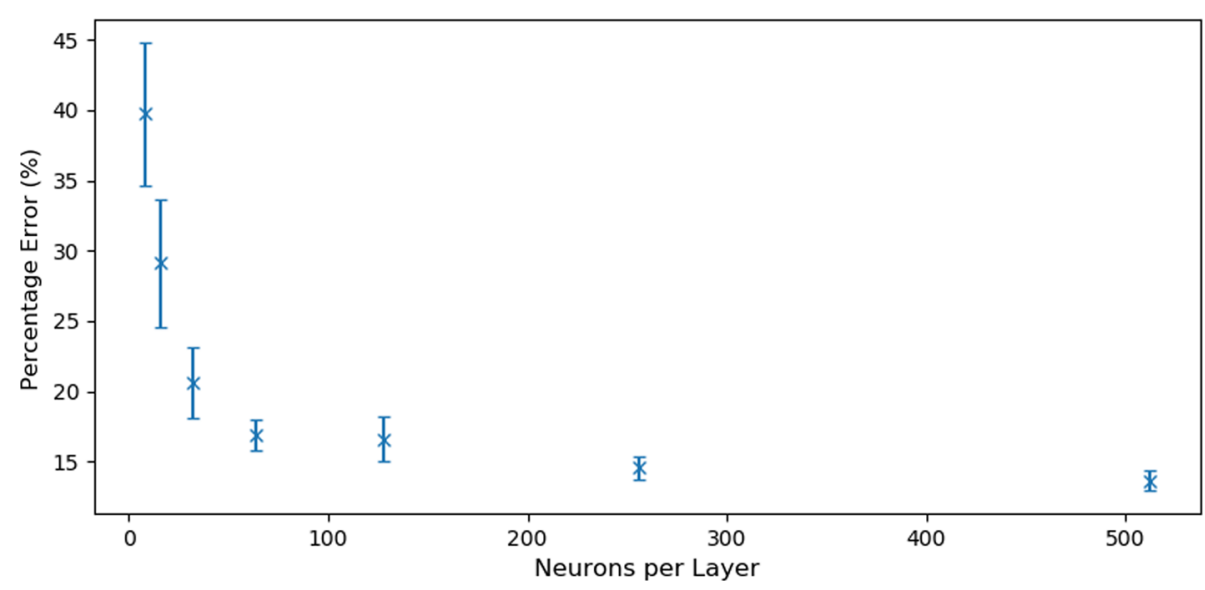

by nature (Pršić et al. 2017; Stojanovic et al. 2016). However, once trained, interrogating the neural network (to retrieve crown height and dimple depth predictions) only took $0.77 \mathrm{~ms}$ per parameter combination, and hence a detailed parameter scan, of $50 * 50 * 50=125,000$ predictions, took less than $100 \mathrm{~s}$. While this method is quick and effective it will not find a true optimum, only the best out of the sampled positions. Indeed, no method which is not explicitly equationbased will be able to find a true optimum position in a fully continuous parameter space, as a better solution with higher precision could always be found. However, in the examined experiment, the parameter space is not truly continuous as, for example, the number of pulses used must be an integer. Even seemingly continuous parameters such as pulse energy have a degree of uncertainty and so the network could indeed be used to find an optimal combination to that precision.

The parameter scan was performed over the full range of each parameter as described in Table 1: laser pulse energy from $5 \mu \mathrm{J}$ to 50 , number of pulses from 50 to 400 , and the $\mu \mathrm{J}$ repetition rate from 10 to $1200 \mathrm{kHz}$. Of these predictions, the parameter combination that yielded the smallest crown height but still satisfied the minimum dimple depth of $4 \mu \mathrm{m}$ was determined to be optimal (best). Table 3 shows this optimal prediction, along with the three best experimental data values in the available data set. The table shows that in all cases the repetition rate is kept at or close to the maximum allowed value of $1200 \mathrm{kHz}$. A similar condition can be seen with the number of pulses where all bar one of the optimal experimental results use 400 pulses. The only result not following this trend is the $3^{\text {rd }}$ best experimental data point. This result shows that a similar crown height can be achieved by reducing the repetition rate, the number of pulses and the pulse energy, although this also results in low dimple depth. Pulse energy in contrast shows a distinct trend with greater pulse energy leading to increased crown height.

As the accuracy of the network has been shown in Table 2, further predictions could be made on the relationship between parameters that fall outside the combinations taken dur- ing experimentation. The predicted trade-off between crown height and number of pulses (Fig. 6a) is particularly interesting as fewer pulses with a constant repetition rate equates to shorter machining times and hence to lower fabrication costs. As the number of pulses increases, the minimum crown height decreases approximately linearly down to around 360 pulses. Beyond this point there seems to be no further improvement. This data makes it easy to determine how the machining time affects the quality of the machined dimple and could prevent unnecessarily long machining times with diminishing returns in terms of part quality.

Each data point in Fig. $6 \mathrm{~b}$ was produced by fixing the number of pulses to a particular value and then querying the $\mathrm{NN}$ for the optimal combination of the remaining parameters that would provide the crown height shown in Fig. 6 a. It was found that, as the number of pulses is reduced, the NN generally predicts that pulse energy must be increased. This gradient is shallowest for high numbers of pulses where the repetition rate is increased to compensate. This occurs until 350 pulses where the repetition rate cannot be further increased and improvements in crown height are also seen to end. The pulse energy needed for low numbers of pulses is double that for high numbers while the repetition rate stays within $20 \%$ of the maximum. To reduce machining time, high repetition rates are desired along with low numbers of pulses, however the repetition rate is already at its maximum for many positions, and where it is lower the effect is smaller than that cause by reducing number of pulses.

\section{Predicted relationship between parameters}

Neural networks can help us to understand the relationships between parameters. From the NN predictions, Fig. 7 shows the effect of changing a single parameter, whilst holding the other two parameters constant at values corresponding to the optimal prediction in Table 1. Figure 7 therefore shows dimple depth and crown height change for varying (a) rep- 
Table 3 Optimal (best) parameters predicted by the neural network compared to experimental data values that had the lowest crown while maintaining a dimple depth of $4 \mu \mathrm{m}$

\begin{tabular}{llclll}
\hline & Repetition rate $(\mathrm{kHz})$ & Pulse energy $(\mu \mathrm{J})$ & Number of pulses & Dimple depth $(\mu \mathrm{m})$ & Crown height $(\mu \mathrm{m})$ \\
\hline Prediction by NN & 1200 & 7.62 & 364 & 4 & 1.26 \\
Best from data (1st) & 1200 & 7.58 & 400 & 4.04 & 1.29 \\
Best from data (2nd) & 1200 & 10.1 & 400 & 5.25 & 1.62 \\
Best from data (3rd) & 1000 & 8.91 & 200 & 4.19 & 1.63 \\
Best from data (4th) & 1200 & 12.4 & 400 & 4.44 & 1.74 \\
\hline
\end{tabular}

Fig. 6 The predicted effect of varying the number of laser pulses. a The effect on minimum crown height. b The required pulse energy and repetition rate to maintain the minimum possible crown height corresponding to the same number of pulse in $\mathbf{a}$

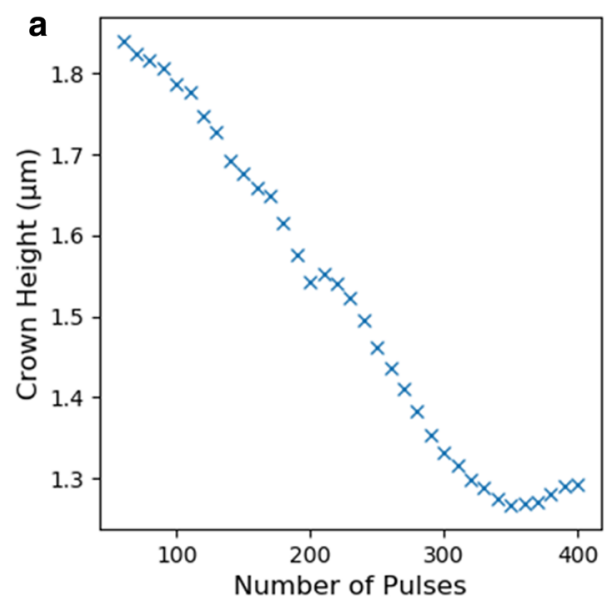

etition rate, (b) pulse energy, and (c) number of pulses. The orange and blue curves correspond to the dimple depth and crown height respectively. The horizontal dotted line shows the $4 \mu \mathrm{m}$ criteria for dimple depth.

Figure 7a shows that increasing pulse energy increases both the dimple depth and crown height, the crown height approximately linearly. The dimple depth however, shows a point of inflexion at $\sim 22 \mu \mathrm{J}$. Clearly, in order to minimise crown height, the pulse energy must take the smallest value possible that still satisfies the $4 \mu \mathrm{m}$ minimum depth (i.e. less than $\sim 10 \mu \mathrm{J})$. Figure $7 \mathrm{~b}$ shows that increasing the number of pulses from $\sim 50$ to $\sim 200$ increases both the dimple depth and the crown height. As the number of pulses exceeds 200, the dimple depth continues to increase whilst the crown height actually decreases. This means that the optimal number of pulses is close to the maximum investigated value. Figure $7 \mathrm{c}$ shows that with low pulse energy and a large number of pulses, repetition rates greater than $\sim 600 \mathrm{kHz}$ are needed in order to produce a dimple depth that is close to the $4 \mu \mathrm{m}$ criteria. To actually meet the $>4 \mu \mathrm{m}$ dimple depth requirement, a repetition rate greater than $1000 \mathrm{kHz}$ is needed. Crown height is approximately constant for repetition rates greater than $\sim 850 \mathrm{kHz}$. Repetition rates less than $\sim 130 \mathrm{kHz}$ produce large crown heights and insufficient depth. There is a sharp increase in dimple depth between $\sim 480$ and $\sim 600 \mathrm{kHz}$. Repetition rates greater than $1000 \mathrm{kHz}$ provide suitable values for both dimple depth and crown height.
In summary, Fig. 6 shows that pulse energy must be minimised, and repetition rate and number of pulses used to compensate. This section demonstrates that neural networks can discern and predict complex patterns of behaviour across multi-dimensioned parameter spaces using only a small number of experimental measurements.

\section{Predictive visualisation of laser machining}

Neural networks also offer the capability for transforming a set of scalar values into a two-dimensional array. Accordingly, here the neural network was adapted in order to transform the three-vector input (pulse energy, number of pulses, and repetition rate) into a two-dimensional array corresponding to the predicted surface profile of the sample after machining with the input parameters. This neural network therefore offered the capability for predictive visualisation of laser machining.

The style of network used was a conditional generative adversarial network (GAN), which uses two competing networks to generate high quality images (I. J. Goodfellow et al. 2014). GANs have been used extensively for a wide range of tasks such as creating faces (Karras et al. 2019) domain-changing image-to-image translation (Grant-Jacob et al. 2019; Isola et al. 2017; Ledig et al. 2017; Ronneberger et al. 2015; Zhu et al. 2017) and even video synthesis (Ma 

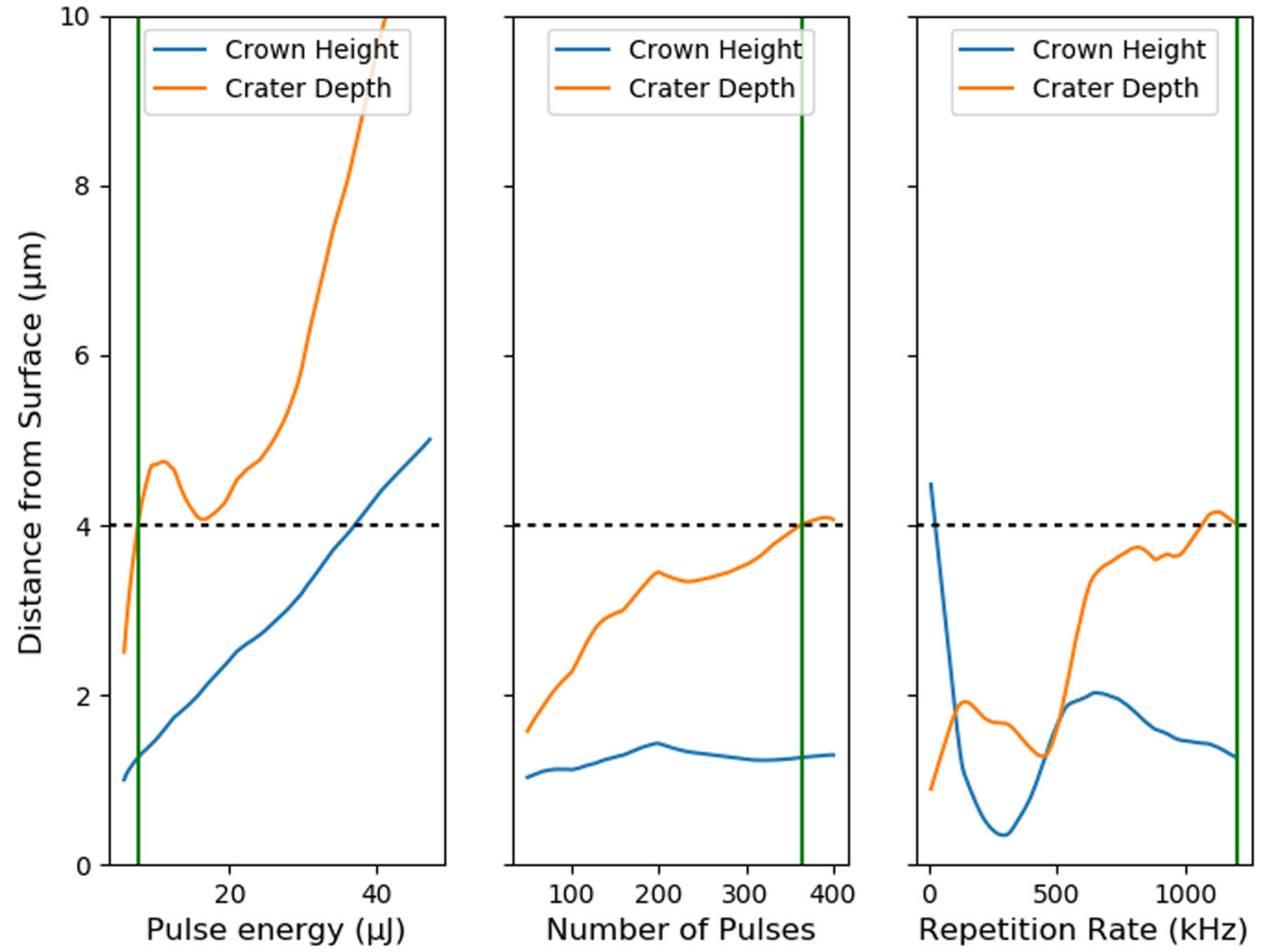

Fig. 7 Neural network predictions for laser machined dimple depth and crown height. a Varying pulse energy. b Varying number of pulses. c Varying repetition rate

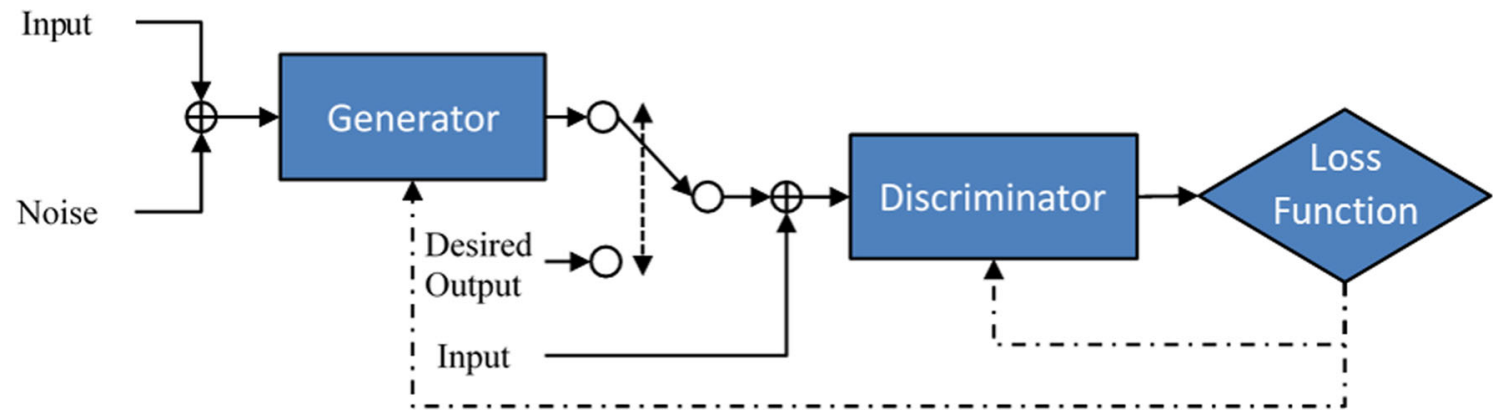

Fig. 8 Schematic of a conditional GAN

et al. 2017; Wang et al. 2018). A schematic showing how conditional GANs work can be seen in Fig. 8 .

The network used in this work was a variation on a progressively growing GAN which starts at low resolution and slowly increases. The input into the generator was the same vector as used for the ANN along with random noise. There was an initial series of fully connected layers to create a latent space from the input, with this being reshaped to form a stack of $2 d$ layers. This was then followed by a series of blocks consisting of two convolution layers and a bicubic up-sampling, with the latent space being up-sampled to be concatenated with each output except the last as shown in Fig. 9. This allows for a flexible network where extra blocks can be added to increase the resolution. Using the network to simulate the laser machining process only takes $14 \mathrm{~ms}$, which shows a clear advantage of using a GAN.

Figure 9 demonstrates the ability of the network to simulate the machining process. Both (a) and (b) are examples of experimentally measured dimple profiles for two different parameter sets, while (c) and (d) are GAN-generated profiles corresponding respectively to the same two sets of machining parameters. Neither of the parameters used for (a) or (b) were 


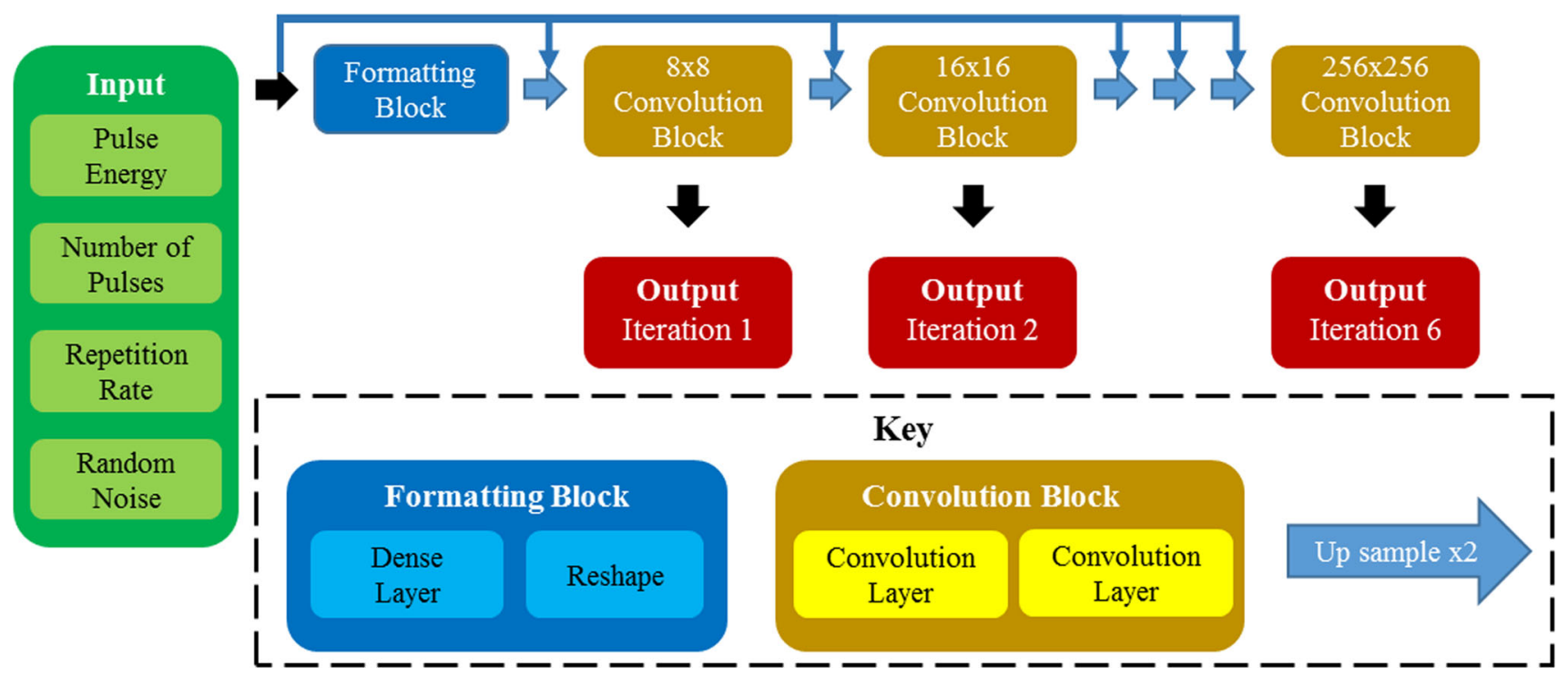

Fig. 9 Block schematic of the GAN generator

included in the training data and so these represent unseen data. The profiles do not match exactly, but that is expected due to the variance in laser machining, even between experimental examples with the same parameters. One area where the GAN falls short, is in the sharpness of the profile. The generated samples are noticeably smoother than the experimental ones and in particular are missing the crown spikes that can be seen in (b). Despite this they are able to match the key parameters of the dimples, with very similar crown height, depth, and diameter. When tested on the same validation combinations used in Fig. 3a and calculating the crown height directly from the results of the GAN, the network had an error of $14.9 \%$, better than both non-NN methods discussed and only slightly worse than the dedicated ANN (Fig. 10).

\section{Comparison between numerical and visualisation techniques}

As discussed in the introduction, conventional optimisation techniques such as gradient search methods and numerical modelling are powerful tools but not suited to all applications. In this work, the complex relationships between parameters means that a gradient search may find a local rather than global minimum, providing misleading results. This problem is also not particularly suited to modelling as a large number of approximations are required, and even then, available computing time is often a limiting factor. These factors provide the motivation for applying NNs for this work.

The two methods discussed in this work have a number of advantages and disadvantages when compared with each other, and each clearly is more suitable for different applica- tions. The biggest advantage of the ANN is its size and speed, with the network used here being trained in 4 min $40 \mathrm{~s}$ on a desktop PC with an Intel Core i7-7700, 32 GB of RAM and an NVidia Titan X GPU. Importantly this time only increased to 5 min $12 \mathrm{~s}$ on a laptop with an Intel Core i5-3210 M 8 GB of RAM and no graphics card. While a high-end consumer graphics card was used in this case, the results could have been duplicated using just a CPU without a large impact on the training time. Interrogation of the network was performed on a CPU demonstrating that specialised hardware was not required for this type of network. ANNs are generally very reliable in producing accurate results with complex data, provided sufficient training data is provided. Best used when a single question needs answering and has numerical input and output but trends are not easy to predict or visualise.

The GAN, on the other hand, took far longer to train and required the use of the high-specification GPU. The same is true for inference where a GPU with comparable RAM would be needed to run the network. They are, however, very powerful tools that can be used to reproduce experiments and their results giving them great flexibility. This is especially apparent when multiple questions must be asked of the network. In this case, for example, if the definition of the crown height was to change, the ANN would have to be completely retrained, while the results of the GAN could be immediately interpreted to provide new the information.

Therefore, where computational time and absolute accuracy of a single result are the main goals of the network, an ANN is likely the preferred choice. However, as discussed above, a GAN has the potential for being a more flexible and comprehensive analytical tool. 

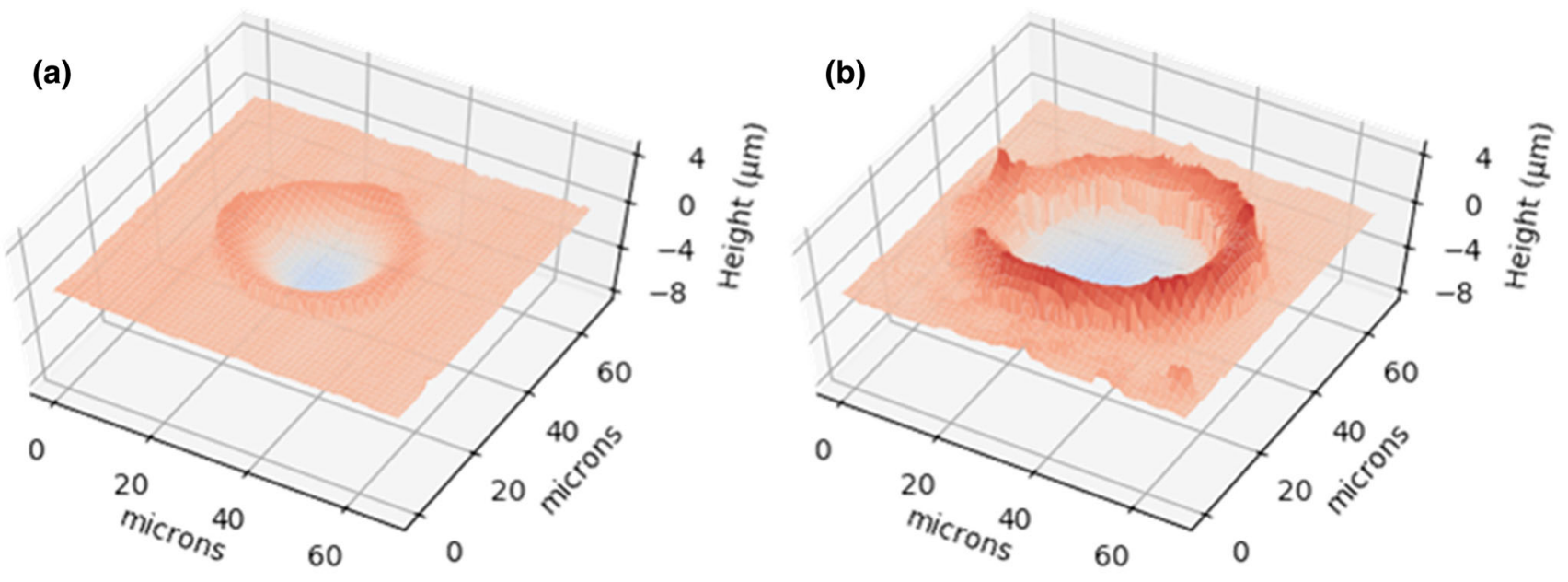

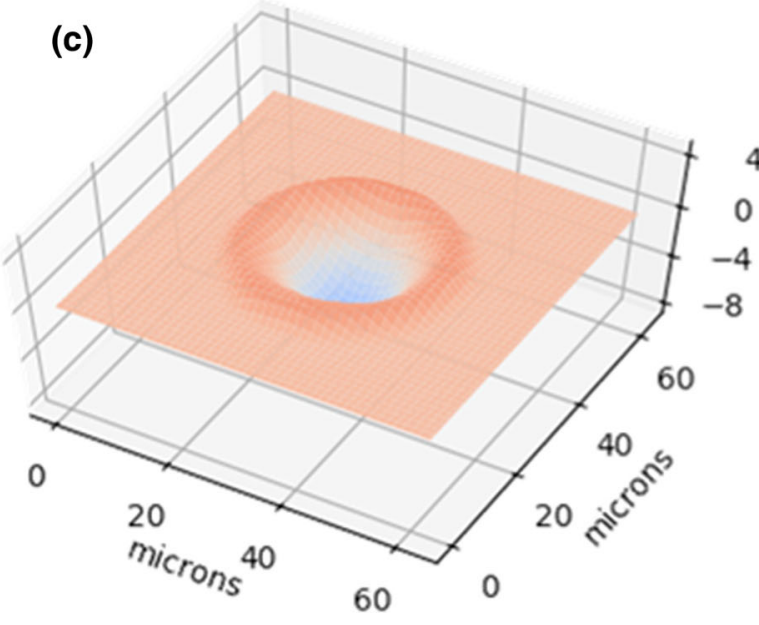

Fig. 10 Visualisation of experimental dimples. a Experimental data machined with a repetition rate of $10 \mathrm{kHz}$, pulse energy $47.17 \mu \mathrm{J}$ and 200 pulses. b Experimental data machined with a repetition rate of

\section{Conclusions}

Neural networks offer the capability for accurate interpolation across multidimensional space, when provided with limited data points. In this work, this principle has been applied to laser machining of blind dimple craters in metallic surfaces for friction reduction, in order to provide a precise optimisation of laser parameters without the need for a systematic experimental exploration of the entire parameter space. Finally, we have demonstrated a GAN that achieves realistic, predictive visualisation of the surface topology of laser machined features directly from numerical data describing the input laser parameters.

In this proof-of-principle demonstration we have developed and examined the methodology for optimising hyperparameters through the specific example of optimising the hyperparameter corresponding to the number of neurons at

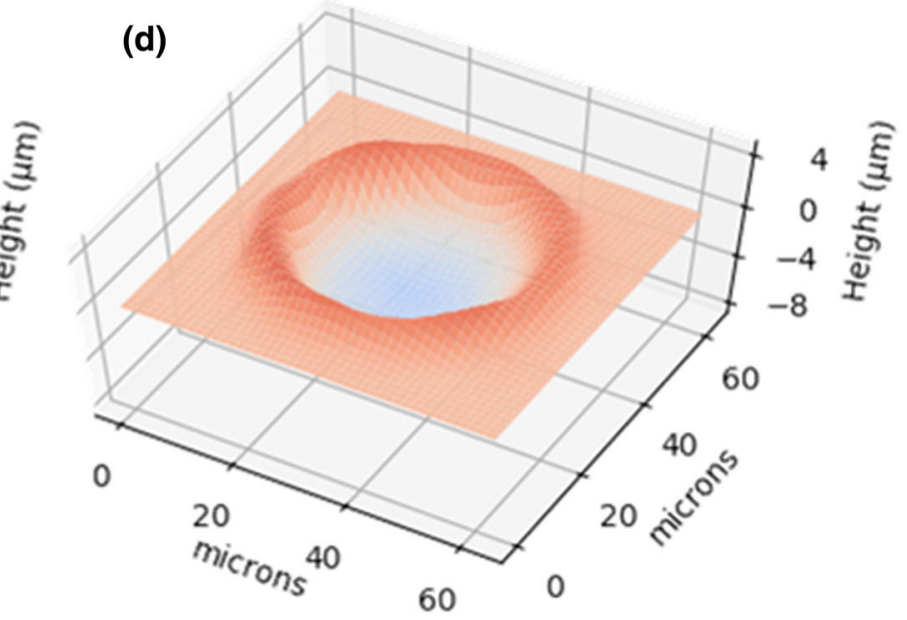

$1200 \mathrm{kHz}$, pulse energy $12.41 \mu \mathrm{J}$ and 100 pulses. c GAN predicted profile with the same machining parameters as (a). d GAN predicted profile with the same machining parameters as (b)

each layer. The techniques demonstrated here can equally be applied to the other hyperparameters, such as the leaning rate, dropout value, and layer specific activation functions. Furthermore, the techniques discussed here could be expanded to include additional experimental parameters such as the pulse duration, wavelength and polarisation. In addition, this proof of principle demonstration could be applied to many other processes across the additive and subtractive laserbased manufacturing application space.

Acknowledgments We gratefully acknowledge the support of NVIDIA Corporation with the donation of the Titan X GPU used for this research. Supporting data for this submission can be found at https://doi.org/1 0.5258/SOTON/D1687. Oxford Lasers acknowledges the support of Innovate UK and Newton Fund (LASTEC Grant Funding No. 102713).

Funding Engineering and Physical Sciences Research Council (EPSRC) (EP/N03368X/1). 
Open Access This article is licensed under a Creative Commons Attribution 4.0 International License, which permits use, sharing, adaptation, distribution and reproduction in any medium or format, as long as you give appropriate credit to the original author(s) and the source, provide a link to the Creative Commons licence, and indicate if changes were made. The images or other third party material in this article are included in the article's Creative Commons licence, unless indicated otherwise in a credit line to the material. If material is not included in the article's Creative Commons licence and your intended use is not permitted by statutory regulation or exceeds the permitted use, you will need to obtain permission directly from the copyright holder. To view a copy of this licence, visit http://creativecomm ons.org/licenses/by/4.0/.

\section{References}

Arnaldo, D., Cerro, D., Pelletier, E., Karnakis, D., Cunha, A., \& Juste, K. (2018). Laser surface texturing of grey cast iron for tribological applications in refrigeration hermetic compressors: the effect of processing parameters on ablated crater rim formation. In The 19th International symposium on laser precision microfabrication.

Asif, K., Zhang, L., Derrible, S., Indacochea, J. E., Ozevin, D., \& Ziebart, B. (2020). Machine learning model to predict welding quality using air-coupled acoustic emission and weld inputs. Journal of Intelligent Manufacturing. https://doi.org/10.1007/s10845020-01667-x.

Campanelli, S. L., Casalino, G., Ludovico, A. D., \& Bonserio, C. (2013). An artificial neural network approach for the control of the laser milling process. International Journal of Advanced Manufacturing Technology, 66(9-12), 1777-1784. https://doi.org/10. 1007/s00170-012-4457-9.

Casalino, G., Losacco, A. M., Arnesano, A., Facchini, F., Pierangeli, M., \& Bonserio, C. (2017). Statistical analysis and modelling of an Yb: KGW femtosecond laser micro-drilling process. Procedia CIRP, 62, 275-280. https://doi.org/10.1016/j.procir.2016.06.111.

Cheng, J., Perrie, W., Edwardson, S. P., Fearon, E., Dearden, G., \& Watkins, K. G. (2009). Effects of laser operating parameters on metals micromachining with ultrafast lasers. Applied Surface Science, 256(5), 1514-1520. https://doi.org/10.1016/j.apsusc.2009. 09.013.

Desiré, M. L., Dijkstra, A., \& Kaufman, L. (1978). Survey of experimental optimization methods (Chap. 11). In D. L. Massart, A. Dijkstra \& L. Kaufman (Eds.), Evaluation and optimization of laboratory methods and analytical procedures (Vol. 1, pp. 213218). Techniques and Instrumentation in Analytical Chemistry. Elsevier. https://doi.org/10.1016/S0167-9244(08)70055-6.

Feng, S., Zhou, H., \& Dong, H. (2019). Using deep neural network with small dataset to predict material defects. Materials and Design, 162, 300-310. https://doi.org/10.1016/j.matdes.2018.11.060.

Gonzalez-Val, C., Pallas, A., Panadeiro, V., \& Rodriguez, A. (2020). A convolutional approach to quality monitoring for laser manufacturing. Journal of Intelligent Manufacturing, 31(3), 789-795. https://doi.org/10.1007/s10845-019-01495-8.

Goodfellow, I., Bengio, Y., \& Courville, A. (2016). Deep learning. Cambridge: MIT Press.

Goodfellow, I. J., Pouget-Abadie, J., Mirza, M., Xu, B., Warde-Farley, D., Ozair, S., et al. (2014). Generative adversarial networks. In Proceedings of the 27th international conference on neural information processing systems (pp. 2672-2680). http://arxiv.org/abs/ 1406.2661.

Grant-Jacob, J. A., Mackay, B. S., Baker, J. A. G., Xie, Y., Heath, D. J., Loxham, M., et al. (2019). A neural lens for super-resolution biological imaging. Journal of Physics Communications, 3(6), 065004. https://doi.org/10.1088/2399-6528/ab267d.
Grant-Jacob, J. A., Mills, B., \& Eason, R. W. (2014). Parametric study of the rapid fabrication of glass nanofoam via femtosecond laser irradiation. Journal of Physics. D. Applied Physics, 47(5), 055105. https://doi.org/10.1088/0022-3727/47/5/055105.

Günther, J., Pilarski, P. M., Helfrich, G., Shen, H., \& Diepold, K. (2014). First steps towards an intelligent laser welding architecture using deep neural networks and reinforcement learning. Procedia Technology, 15, 474-483. https://doi.org/10.1016/j.protcy.2014.09.00 7.

Günther, J., Pilarski, P. M., Helfrich, G., Shen, H., \& Diepold, K. (2016). Intelligent laser welding through representation, prediction, and control learning: An architecture with deep neural networks and reinforcement learning. Mechatronics, 34, 1-11. https://doi.org/1 0.1016/j.mechatronics.2015.09.004.

Heath, D. J., Grant-Jacob, J. A., Eason, R. W., \& Mills, B. (2018a). Single-pulse ablation of multi-depth structures via spatially filtered binary intensity masks. Applied Optics, 57(8), 1904-1909. https://doi.org/10.1364/AO.57.001904.

Heath, D. J., Grant-Jacob, J. A., Xie, Y., Mackay, B. S., Baker, J. A. G., Eason, R. W., et al. (2018b). Machine learning for 3D simulated visualization of laser machining. Optics Express, 26(17), 21574-21584. https://doi.org/10.1364/OE.26.021574.

Isola, P., Zhu, J.-Y., Zhou, T., \& Efros, A. A. (2017). Image-to-image translation with conditional adversarial networks. In 2017 IEEE conference on computer vision and pattern recognition (CVPR) (pp. 5967-5976). IEEE. https://doi.org/10.1109/CVPR.2017.632.

Karras, T., Laine, S., \& Aila, T. (2019). A style-based generator architecture for generative adversarial networks. In arXiv preprint arXiv:1812.04948. http://arxiv.org/abs/1812.04948.

Kim, D. H., Kim, T. J. Y., Wang, X., Kim, M., Quan, Y. J., Oh, J. W., et al. (2018). Smart machining process using machine learning: A review and perspective on machining industry. International Journal of Precision Engineering and Manufacturing-Green Technology, 5(4), 555-568. https://doi.org/10.1007/s40684-0180057-y.

Kingma, D. P., \& Ba, J. (2014). Adam: A method for stochastic optimization. arXiv preprint arXiv:1412.6980. http://arxiv.org/abs/1 412.6980 .

Le Harzic, R., Huot, N., Audouard, E., Jonin, C., Laporte, P., Valette, S., et al. (2002). Comparison of heat-affected zones due to nanosecond and femtosecond laser pulses using transmission electronic microscopy. Applied Physics Letters, 80(21), 3886-3888. https:// doi.org/10.1063/1.1481195.

Ledig, C., Theis, L., Huszar, F., Caballero, J., Cunningham, A., Acosta, A., et al. (2017). Photo-realistic single image superresolution using a generative adversarial network. In 2017 IEEE conference on computer vision and pattern recognition (CVPR) (pp. 105-114). IEEE. https://doi.org/10.1109/CVPR.2017.19.

Li, X., Jia, X., Yang, Q., \& Lee, J. (2020). Quality analysis in metal additive manufacturing with deep learning. Journal of Intelligent Manufacturing. https://doi.org/10.1007/s10845-020-01549-2.

Liu, J. M. (1982). Simple technique for measurements of pulsed Gaussian-beam spot sizes. Optics Letters, 7(5), 196-198. https:// doi.org/10.1364/OL.7.000196.

Lorbeer, R.-A., Pastow, J., Sawannia, M., Klinkenberg, P., Förster, D., \& Eckel, H.-A. (2017). Power spectral density evaluation of laser milled surfaces. Materials, 11(1), 50-60. https://doi.org/10.3390/ ma11010050.

Ma, L., Jia, X., Sun, Q., Schiele, B., Tuytelaars, T., \& Van Gool, L. (2017). Pose Guided Person Image Generation. In 31 st Conference on neural information processing systems. http://arxiv.org/abs/17 05.09368

Mahato, V., Obeidi, M. A., Brabazon, D., \& Cunningham, P. (2020). Detecting voids in 3D printing using melt pool time series data. Journal of Intelligent Manufacturing. https://doi.org/10.1007/s10 845-020-01694-8. 
Mazhukin, V. I. (2017). Nanosecond laser ablation: Mathematical models, computational algorithms, Modeling. In Laser ablation-From fundamentals to applications (pp. 31-55). IntechOpen. https://doi.org/10.5772/intechopen.70773.

Mezzapesa, F. P., Scaraggi, M., Carbone, G., Sorgente, D., Ancona, A., \& Lugarà, P. M. (2013). Varying the geometry of laser surface microtexturing to enhance the frictional behavior of lubricated steel surfaces. Physics Procedia, 41, 677-682. https://doi.org/10. 1016/j.phpro.2013.03.132.

Mills, B., Heath, D. J., Feinaeugle, M., Grant-Jacob, J. A., \& Eason, R. W. (2014). Laser ablation via programmable image projection for submicron dimension machining in diamond. Journal of Laser Applications, 26(4), 041501. https://doi.org/10.2351/1.4893749.

Mills, B., Heath, D. J., Grant-Jacob, J. A., \& Eason, R. W. (2018). Predictive capabilities for laser machining via a neural network. Optics Express, 26(13), 17245-17253. https://doi.org/10.1364/ OE.26.017245.

Misawa, H., Sun, H.-B., Juodkazis, S., Watanabe, M., \& Matsuo, S. (2000). Microfabrication by femtosecond laser irradiation. In H. Helvajian, K. Sugioka, M. C. Gower, \& J. J. Dubowski (Eds.), Laser Applications in Microelectronic and Optoelectronic Manufacturing $V$ (Vol. 3933, pp. 246-260). SPIE. https://doi.org/10.1 $117 / 12.387561$

Mittal, S., Khan, M. A., Romero, D., \& Wuest, T. (2019). Smart manufacturing: Characteristics, technologies and enabling factors. Proceedings of the Institution of Mechanical Engineers, Part B: Journal of Engineering Manufacture, 233(5), 1342-1361. https:// doi.org/10.1177/0954405417736547.

Momma, C., Nolte, S., Chichkov, B. N., Alvensleben, F., \& Tünnermann, A. (1997). Precise laser ablation with ultrashort pulses. Applied Surface Science, 109-110, 15-19. https://doi.org/10.101 6/S0169-4332(96)00613-7.

Mycroft, W., Katzman, M., Tammas-Williams, S., Hernandez-Nava, E., Panoutsos, G., Todd, I., et al. (2020). A data-driven approach for predicting printability in metal additive manufacturing processes. Journal of Intelligent Manufacturing, 31(7), 1769-1781. https:// doi.org/10.1007/s10845-020-01541-w.

Neuenschwander, B., Jaeggi, B., \& Schmid, M. (2013). From fs to Subns: Dependence of the material removal rate on the pulse duration for metals. Physics Procedia, 41, 794-801. https://doi.org/10.10 16/j.phpro.2013.03.150.

Oloyede, O., Bigg, T. D., Cochrane, R. F., \& Mullis, A. M. (2016). Microstructure evolution and mechanical properties of drop-tube processed, rapidly solidified grey cast iron. Materials Science and Engineering A, 654, 143-150. https://doi.org/10.1016/j.msea.201 5.12.020.

Otto, A., Koch, H., \& Vazquez, R. G. (2012). Multiphysical simulation of laser material processing. Physics Procedia, 39(13), 843-852. https://doi.org/10.1016/j.phpro.2012.10.109.

Penumuru, D. P., Muthuswamy, S., \& Karumbu, P. (2020). Identification and classification of materials using machine vision and machine learning in the context of industry 4.0. Journal of Intelligent Manufacturing, 31(5), 1229-1241. https://doi.org/10.1007/ s10845-019-01508-6.

Pršić, D., Nedić, N., \& Stojanović, V. (2017). A nature inspired optimal control of pneumatic-driven parallel robot platform. Proceedings of the Institution of Mechanical Engineers, Part C: Journal of Mechanical Engineering Science, 231(1), 59-71. https://doi.org/ 10.1177/0954406216662367.

Qu, N., Chen, X., Li, H., \& Zeng, Y. (2014). Electrochemical micromachining of micro-dimple arrays on cylindrical inner surfaces using a dry-film photoresist. Chinese Journal of Aeronautics, 27(4), 1030-1036. https://doi.org/10.1016/j.cja.2014.03.012.

Ren, J., Kelly, M., \& Hesselink, L. (2005). Laser ablation of silicon in water with nanosecond and femtosecond pulses. Optics Letters, 30(13), 1740-1742. https://doi.org/10.1364/OL.30.001740.
Ronneberger O., Fischer P., Brox T. (2015) U-Net: Convolutional networks for biomedical image segmentation. In N. Navab, J. Hornegger, W. Wells, A. Frangi (Eds.) Medical image computing and computer-assisted intervention-MICCAI 2015. Lecture Notes in Computer Science (Vol. 9351). Cham: Springer. https:// doi.org/10.1007/978-3-319-24574-4_28.

Sakai, T., Nedyalkov, N., \& Obara, M. (2007). Friction characteristics of submicrometre-structured surfaces fabricated by particleassisted near-field enhancement with femtosecond laser. Journal of Physics. D. Applied Physics, 40(23), 7485-7491. https://doi. org/10.1088/0022-3727/40/23/035.

Scaraggi, M., Mezzapesa, F. P., Carbone, G., Ancona, A., Sorgente, D., \& Lugarà, P. M. (2014). Minimize friction of lubricated lasermicrotextured-surfaces by tuning microholes depth. Tribology International, 75, 123-127. https://doi.org/10.1016/j.triboint.20 14.03.014.

Simonyan, K., \& Zisserman, A. (2015). Very deep convolutional networks for large-scale image recognition. In Very deep convolutional networks for large-scale image recognition ICLR2015.

Stegemann, J. A., \& Buenfeld, N. R. (1999). A glossary of basic neural network terminology for regression problems. Neural Computing and Applications, 8(4), 290-296. https://doi.org/10.1007/s00521 0050034.

Stojanovic, V., \& Filipovic, V. (2014). Adaptive input design for identification of output error model with constrained output. Circuits, Systems, and Signal Processing, 33(1), 97-113. https://doi.org/1 0.1007/s00034-013-9633-0.

Stojanovic, V., \& Nedic, N. (2016). Identification of time-varying OE models in presence of non-Gaussian noise: Application to pneumatic servo drives. International Journal of Robust and Nonlinear Control, 26(18), 3974-3995. https://doi.org/10.1002/rnc.3544.

Stojanovic, V., Nedic, N., Prsic, D., Dubonjic, L., \& Djordjevic, V. (2016). Application of cuckoo search algorithm to constrained control problem of a parallel robot platform. The International Journal of Advanced Manufacturing Technology, 87(9-12), 2497-2507. https://doi.org/10.1007/s00170-016-8627-z.

Teixidor, D., Grzenda, M., Bustillo, A., \& Ciurana, J. (2015). Modeling pulsed laser micromachining of micro geometries using machinelearning techniques. Journal of Intelligent Manufacturing, 26(4), 801-814. https://doi.org/10.1007/s10845-013-0835-x.

Wang, T.-C., Liu, M.-Y., Zhu, J.-Y., Liu, G., Tao, A., Kautz, J., \& Catanzaro, B. (2018). Video-to-Video Synthesis. In Proceedings of the 32 nd international conference on neural information processing systems (pp. 1152-1164). http://arxiv.org/abs/1808.06601.

Werbos, P. (1974). Beyond regression: New tools for prediction and analysis in the behavioral sciences. Harvard: Harvard University.

Yousef, B. F., Knopf, G. K., Bordatchev, E. V., \& Nikumb, S. K. (2003). Neural network modeling and analysis of the material removal process during laser machining. The International Journal of Advanced Manufacturing Technology, 22(1-2), 41-53. https://doi. org/10.1007/s00170-002-1441-9.

Zahavy, T., Dikopoltsev, A., Moss, D., Haham, G. I., Cohen, O., Mannor, S., et al. (2018). Deep learning reconstruction of ultrashort pulses. Optica, 5(5), 666-673. https://doi.org/10.1364/OPTICA. 5.000666.

Zhang, Y., \& Ling, C. (2018). A strategy to apply machine learning to small datasets in materials science. NPJ Computational Materials, 4(1), 25-32. https://doi.org/10.1038/s41524-018-0081-z.

Zhu, J. -Y., Park, T., Isola, P., \& Efros, A. A. (2017). Unpaired Image-toImage Translation Using Cycle-Consistent Adversarial Networks. In 2017 IEEE international conference on computer vision (ICCV) (pp. 2242-2251). IEEE. https://doi.org/10.1109/ICCV.2017.244.

Publisher's Note Springer Nature remains neutral with regard to jurisdictional claims in published maps and institutional affiliations. 